\title{
Actividad física y consejería en estudiantes universitarios de primero y quinto año de medicina de Bogotá, Colombia
}

\author{
Physical activity and counseling in students of first and fifth year of medicine course in one university of Bogotá, \\ Colombia
}

\author{
Gustavo Tovar' ${ }^{1}$ Ángela Rodríguez², Gabriela García ${ }^{3}$, José Rafael Tovar ${ }^{4}$
}

\author{
1 Médico. Profesor Escuela de Medicina y Ciencias de la Salud. Universidad del Rosario. Bogotá, Colombia. e-mail: \\ gustavo.tovar@urosario.edu.co \\ 2 Fisioterapeuta. Universidad del Rosario. Bogotá, Colombia. e-mail: angela.m1505@gmail.com \\ 3 Fisioterapeuta. Universidad del Rosario. Bogotá, Colombia. e-mail: gainyi@gmail.com \\ 4 Estadístico. PhD. Escuela de Estadística Universidad del Valle, Cali, Colombia. e-mail: jose.r.tovar@correounivalle.edu.co
}

\section{Resumen}

Introducción: La inactividad física es el cuarto factor de riesgo para desarrollar una enfermedad no transmisible. La asesoría brindada por el médico depende de sus propios hábitos de vida. Objetivo: Describir los niveles de actividad física y los factores relacionados con la consejería en estudiantes de primero y quinto año de la facultad de medicina de una Universidad privada en Bogotá, Colombia. Materiales y métodos: Estudio descriptivo con 61 estudiantes de primer año y 62 de quinto año de medicina con edades comprendidas entre 18 y 29 años. Se analizaron los datos de un subconjunto de registros, que hacen parte de un archivo mayor de datos obtenido en un estudio de corte transversal, en el que se aplicó la versión en español del cuestionario Healthy Doctor $=$ Healthy Patient. Resultados: Se evaluaron 123 estudiantes de primero y quinto año de medicina. En primer año el 39,3\% y en quinto año el 48,8\% cumplieron con las recomendaciones de actividad física. Ser inactivo lo reportaron el $21,3 \%$ y el $8,1 \%$ respectivamente. La falta de tiempo fue la principal barrera para realizar actividad física. La actitud positiva hacia la consejería fue del $100 \%$ en el último año. Se observó más sobrepeso en el grupo de quinto año (23,8\% hombres y $9.8 \%$ mujeres). Conclusión: La proporción de estudiantes de medicina que realizan actividad física es inferior a la observada en la población general y en poblaciones similares de estudiantes de medicina. El análisis de las barreras sugiere que en futuras intervenciones se debe pensar en la adjudicación de un tiempo para su práctica dentro del currículo. Se debe repensar la forma en que las facultades de medicina están abordando el tema de la actividad física, como pilar fundamental de la promoción de la salud.

Palabras clave: Actividad física; actitud frente a la salud; estudiantes de medicina; estilo de vida; escuelas médicas; medicina preventiva. (Fuente: DeCS, Bireme).

\begin{abstract}
Introduction: Physical inactivity is the fourth risk factor for developing a Non transmissible Chronic Disease (NCD). The advice given by the physician depends on people's own lifestyle. Objective: To describe the levels of physical activity (PA) and factors associated with counseling in students from the first and fifth year of the faculty of medicine at a private university in Bogotá, Colombia. Materials and methods: A descriptive study was made with 61 first-year
\end{abstract}


students and 62 from fifth year of medicine between 18 and 29 years old. The data from a subset of records was analyzed, which are part of a database obtained in a cross-sectional study, in which the Spanish version of the questionnaire Healthy Doctor = Healthy patient was applied. Results: 123 first and fifth year of medicine students were evaluated. In first year 39.3\% and in fifth year 48.8\% met the recommendations of PA. $21.3 \%$ and $8.1 \%$ respectively reported to be inactive. Lack of time was the main barrier to physical activity. Positive attitude toward counseling was $100 \%$ in the last year. More overweight was observed in the group of fifth year $(23.8 \%$ men and $9.8 \%$ women). Conclusion: The proportion of medical students who perform PA is lower than those observed in the general population and in similar populations of medicine students. The analysis of the barriers suggests that future interventions should consider the allocation of time to practice it within the curriculum. The way how medical schools are addressing the issue of physical activity must be reviewed as a fundamental pillar of health promotion.

Keywords: Physical activity; attitude towards health; medicine students; lifestyle; schools medical schools; preventive medicine. (Source: DeCS, Bireme).

\section{Introducción}

La actividad física (AF) es uno de los pilares clave para la prevención de las enfermedades no transmisibles (ENT), su práctica regular es un factor protector para la prevención y el manejo de enfermedades cardiovasculares, metabólicas y para algunos tipos de cáncer.1,2 Por otro lado, la inactividad física se ha convertido en uno de los problemas más prevalentes de salud pública de la población mundial.2,3

De acuerdo con datos de la Organización Mundial de la Salud (OMS), la inactividad física es el cuarto factor de riesgo para desarrollar una ENT, en el 2010 más de 3 millones de muertes fueron asociadas a este factor. La actividad física constituye un factor protector contra el estrés oxidativo, se ha observado en las ENT un desbalance entre el ingreso (ingesta de alimento) y la salida (gasto energético), esta es una de las razones para considerar inactividad física como un factor determinante para la aparición de dichas enfermedades.2,3

$\mathrm{Al}$ analizar el panorama mundial el $68,9 \%$ de la población presenta un nivel de actividad física muy por debajo de lo recomendado "150 minutos a la semana de intensidad moderada-vigorosa para adultos". En Colombia no alcanzaron los niveles de actividad recomendados el $42.7 \%$; $38.1 \%$ en hombres y $47.1 \%$ en mujeres. 4,5

Estudios epidemiológicos han determinado que la inactividad física aumenta entre 20 y $30 \%$, el riesgo para desarrollar una ENT; adicional a esto, la OMS revela que por cada $10 \%$ de aumento en la cifras de inactividad hay una disminución del $0.5 \%$ del crecimiento económico anual de un país.4,6

Para hacer frente a esta situación se han propuesto diversas acciones, una de las más promulgadas es la promoción de la salud. En esta línea se han realizado varias investigaciones acerca de la consejería en salud aplicada por el personal médico, las cuales han demostrado que existe una asociación positiva entre la asesoría brindada por el médico y cambios significativos en los hábitos de vida de los pacientes.7,8

Varias investigaciones han reportado que en general, los médicos le expresan a sus pacientes la necesidad de practicar AF, sin embargo, se ha detectado vacíos al momento de dar indicaciones específicas acerca del tipo de actividad, frecuencia e intensidad que se requiere para obtener los beneficios deseados.9,10

Las investigaciones sobre el tema de la consejería se han centrado en la práctica de hábitos saludables por parte del profesional o el estudiante de medicina, la presencia de temáticas de consejería y promoción de la salud dentro del currículo, el fomento de hábitos saludables al interior de las facultades y la falta de acceso o tiempo para la práctica de AF y se ha encontrado relación con la probabilidad de que el médico realice $o$ no consejería en salud a sus pacientes. 11,12

Investigaciones realizadas con estudiantes de medicina en varios países de América Latina y en los Estados Unidos evidencian prevalencias de sedentarismo que van del $46 \%$ en Estados Unidos 
al 70\% en Chile.13,14 En Colombia, se tomó como modelo el cuestionario "Healthy Doctor = Healthy Patient" y posterior a su adaptación cultural, se aplicó a ocho facultades de medicina ubicadas en Bogotá. Se encontró que el $50 \%$ de los estudiantes cumplen con la recomendación de AF y presentan un grado mínimo de conocimiento de las recomendaciones para la prescripción de la misma.15,16

Por tal razón, se planteó este estudio con el objetivo de describir los niveles de actividad física y la presencia de factores relacionados con la práctica y consejería de la misma, en estudiantes de primer y quinto año de la Facultad de medicina, a fin de presentar líneas de base que permitan sugerir cambios en la forma en que se aborda el tema de la actividad física dentro del pensum.

\section{Materiales y métodos}

El estudio fue desarrollado con la información contenida en un subconjunto de registros parte de un archivo de datos mayor obtenido en un estudio de corte transversal en el que se aplicó la versión en español del cuestionario Healthy Doctor = Healthy Patient. En dicho estudio se seleccionó un número de instituciones universitarias a las que el grupo de investigadores tenía fácil acceso (muestreo por conveniencia) y se seleccionaron grupos de estudiantes matriculados en segundo (primer año) y noveno (último año) semestre de la carrera de medicina. En el marco de ese proyecto, se sometió el instrumento a un proceso de traducción - retraducción y adaptación cultural, con la obtención posterior de los índices de validez y confiabilidad.

Para la realización del presente estudio, se hizo un análisis detallado de las 65 preguntas del cuestionario y con base en la revisión de la literatura, se decidió que las respuestas a 41 de las mismas permitían tener la información suficiente para cumplir con los objetivos propuestos. Se tomaron los registros cribados por investigadores de una de las universidades participantes en el estudio mayor; de modo que se contó con 61 registros de individuos matriculados en primer año y 62 registros de matriculados en quinto año de medicina.13,15

Se tomó información de variables como el género, el año de carrera en estudio, el estrato socioeconómico categorizado como alto (estratos 5 y 6), medio (estratos 3 y 4) y bajo (estratos 1 y 2); la percepción del estado de salud categorizado como excelente, muy bueno, bueno, regular y malo. Se contó también con información del índice de masa corporal (IMC), la cantidad de actividad física realizada medida en minutos, aspectos que el estudiante consideraba barreras y facilitadores para la realización de $\mathrm{AF}$, la actitud hacia la promoción de AF y conocimiento sobre las recomendaciones de $\mathrm{AF}$.

El IMC se calculó a partir del peso y la talla reportados por los participantes y se categorizó en infrapeso $\left(<18,5 \mathrm{Kg} / \mathrm{m}^{2}\right)$, peso normal $(18,5$ $24,9 \mathrm{Kg} / \mathrm{m}^{2}$ ) y sobrepeso y obesidad $(\geq 25$ $\mathrm{Kg} / \mathrm{m}^{2}$ ). Se clasificó a los participantes en cuatro grupos de acuerdo con el cumplimiento de las recomendaciones de actividad física a saber: inactivo los que reportaron realizar cero (0) minutos (min) de AFM o de AFV a la semana, nivel bajo a los que realizaban $<150$ minutos combinados de AFM y AFV, nivel medio a los que realizaban de 150 a 300 minutos combinados de AFM y AFV, y nivel alto aquellos que realizaban más de 300 minutos combinados de AFM y AFV por semana.17

El estudio también recabó datos auto reportados acerca de la presencia antecedentes familiares y/o personales de: diabetes, hipertensión, hipercolesterolemia, derrame cerebral, obesidad, tabaquismo, dependencia de alcohol o algún tipo de cáncer los cuales no eran excluyentes entre sí, de tal manera que no se discriminó que miembro de la familia presentaba dicho factor de riesgo. Para evaluar los aspectos considerados barreras y facilitadores la encuesta presentaba una escala Likert en las que el individuo debía reportar su grado de acuerdo o desacuerdo, acerca de aspectos como falta de tiempo, falta de apoyo social, falta de energía vital para realizar actividad física, falta de recursos económicos, falta de habilidades o miedo a lesionarse, la formación académica y el apoyo institucional en la promoción de hábitos saludables. 
En cuanto a las preguntas relacionadas con la actitud hacia la consejería en salud, se indagó por aspectos como: la realización de actividad física, la relación entre estar en buen estado y la capacidad para realizar consejería en salud, además si consideraba relevante aconsejar a los pacientes acerca de AF/ejercicio. El último bloque de preguntas iba dirigido a establecer el nivel de conocimiento de los estudiantes acerca de las recomendaciones mundiales en $\mathrm{AF}$, la frecuencia, intensidad y duración del ejercicio y el cómo calcular la frecuencia cardiaca máxima. Se clasificó como ausencia de conocimiento si ninguna respuesta era correcta.

El archivo de datos usado para el procesamiento estadístico contaba con un código de identificación de modo que no se expuso la información de identificación de los estudiantes. El proyecto marco contó con la aprobación del Comité de Ética Médica de la Universidad del Rosario.

El análisis de datos se realizó con la ayuda del paquete estadístico SPSS versión 21 para Windows y se empleó la distribución de frecuencia simples y tablas de contingencia.

\section{Resultados}

La muestra de estudio estuvo conformada por 123 estudiantes, (49,6\% primer año y 50,4\% quinto año) en ambos grupos más del $60 \%$ de los participantes eran mujeres de edades promedio entre 18 y 29 años. En la distribución según el estrato socioeconómico, el $65 \%$ se ubicó en estrato medio. La mayoría de los sujetos presentaron un peso considerado normal de acuerdo con el IMC (el 77\% de los estudiantes de primer año y un 80,6\% de los de quinto año), sin embargo, por género se observan diferencias, en el grupo de primer año los hombres con peso normal eran el $80 \%$ (75,6\% de las mujeres) y en el grupo de primer año, el $66,7 \%$ de los hombres presentaba un peso normal $(87,8 \%$ de las mujeres), se observó una proporción mayor de individuos con sobrepeso y obesidad en el grupo de quinto año.

En el grupo de estudiantes de primer año, el antecedente factor de riesgo con mayor presencia fue el tabaquismo (41\%), seguido por la hipercolesterolemia $(34,1 \%)$. En los estudiante de quito año el factor de riesgo con mayor reporte fue la hipercolesterolemia $(56,5 \%)$ seguido por el tabaquismo con un 53,2\%. (Tabla 1).

Tabla 1. Índice de masa corporal (IMC) y antecedentes familiares de factores de riesgo cardiovascular por sexo en los dos grupos de estudiantes de medicina

\begin{tabular}{|c|c|c|c|c|c|c|c|}
\hline \multirow{2}{*}{\multicolumn{2}{|c|}{ Característica }} & \multicolumn{3}{|c|}{$\begin{array}{c}\text { Primer Año } \\
(n=61) 49,6 \% \\
\text { Proporción según sexo }\end{array}$} & \multicolumn{3}{|c|}{$\begin{array}{c}\text { Quinto Año } \\
\text { (n=62) 50,4 \% } \\
\text { Proporción según sexo }\end{array}$} \\
\hline & & $\begin{array}{r}\text { Hombres } \\
n=20 \\
32,8 \% \\
\end{array}$ & $\begin{array}{r}\text { Mujeres } \\
n=41 \\
67,2 \% \\
\end{array}$ & $\begin{array}{l}\text { Total } \\
n=61\end{array}$ & $\begin{array}{r}\text { Hombres } \\
\mathbf{n}=21 \\
33,9 \% \\
\end{array}$ & $\begin{array}{r}\text { Mujeres } \\
n=41 \\
66,1 \% \\
\end{array}$ & $\begin{array}{l}\text { Total } \\
\qquad n=62\end{array}$ \\
\hline \multirow{6}{*}{ IMC (Índice de masa corporal) } & Bajo peso & $15 \%$ & $19,5 \%$ & $18 \%$ & $9,5 \%$ & $2,4 \%$ & $4,8 \%$ \\
\hline & Normal & $80 \%$ & $75,6 \%$ & $77 \%$ & $66,7 \%$ & $87,8 \%$ & $80,6 \%$ \\
\hline & Sobrepeso y obesidad & $5 \%$ & $4,9 \%$ & $4,9 \%$ & $23,8 \%$ & $9,8 \%$ & $14,5 \%$ \\
\hline & Diabetes & $0 \%$ & $4,9 \%$ & $3,3 \%$ & $9,5 \%$ & $9,8 \%$ & $9,7 \%$ \\
\hline & Hipertensión arterial & $30 \%$ & $26,8 \%$ & $27,9 \%$ & $28,6 \%$ & $34,1 \%$ & $32,3 \%$ \\
\hline & Hipercolesterolemia & $25 \%$ & $34,1 \%$ & $31,1 \%$ & $42,9 \%$ & $63,4 \%$ & $56,5 \%$ \\
\hline \multirow{5}{*}{ Factores de Riesgo } & Obesidad & $25 \%$ & $9,8 \%$ & $14,8 \%$ & $23,8 \%$ & $22 \%$ & $22,6 \%$ \\
\hline & Tabaquismo & $40 \%$ & $41,5 \%$ & $41 \%$ & $57,1 \%$ & $51,2 \%$ & $53,2 \%$ \\
\hline & Alcoholismo & $20 \%$ & $4,9 \%$ & $9,8 \%$ & $4,8 \%$ & $17,1 \%$ & $12,9 \%$ \\
\hline & Cáncer & $0 \%$ & $7,3 \%$ & $4,9 \%$ & $14,3 \%$ & $9,8 \%$ & $11,3 \%$ \\
\hline & Derrame cerebral & $5 \%$ & $12,2 \%$ & $9,8 \%$ & $0 \%$ & $7,3 \%$ & $4,8 \%$ \\
\hline
\end{tabular}


Respecto a las barreras y facilitadores para la realización de AF, la principal causa reportada en ambos grupos fue la falta de tiempo. Frente a la actitud hacia la promoción, la distribución según el género mostró que en primer año el 95\% de los hombres y el $100 \%$ de las mujeres presentaban una actitud favorable hacia la consejería; en quinto año el 100\% de los estudiantes presentó una actitud positiva. Es importante destacar la diferencia frente al conocimiento que se tiene sobre las recomendaciones de actividad física, se observó que el 2,6 \% de los estudiantes de primer año respondió erróneamente las preguntas y 3,6 de los estudiante de quito año fallaron en las mismas preguntas. (Tabla 2).

Tabla 2. Distribución de barreras, facilitadores, actitud y conocimientos para realizar actividad física por sexo en los dos grupos de estudiantes

\begin{tabular}{|c|c|c|c|c|c|c|c|}
\hline \multirow{4}{*}{ Característica } & & \multirow{2}{*}{\multicolumn{3}{|c|}{$\begin{array}{c}\text { Primer Año } \\
(n=61) 49,6 \% \\
\text { Proporción según sexo }\end{array}$}} & \multirow{2}{*}{\multicolumn{3}{|c|}{$\begin{array}{c}\text { Quinto Año } \\
(n=62) 50,4 \% \\
\text { Proporción según sexo }\end{array}$}} \\
\hline & & & & & & & \\
\hline & & \multirow{2}{*}{$\begin{array}{r}\text { Hombres } \\
\mathrm{n}=\mathbf{2 0} \\
\mathbf{3 2 , 8 \%} \\
\end{array}$} & \multirow{2}{*}{$\begin{array}{r}\text { Mujeres } \\
\mathrm{n}=41 \\
67,2 \% \\
\end{array}$} & \multirow{2}{*}{${ }_{n=61}^{\text {Total }}$} & \multirow{2}{*}{$\begin{array}{r}\text { Hombres } \\
\mathbf{n}=21 \\
33,9 \% \\
\end{array}$} & \multirow{2}{*}{$\begin{array}{r}\text { Mujeres } \\
\mathrm{n}=41 \\
66,1 \% \\
\end{array}$} & \multirow{2}{*}{$\begin{array}{l}\text { Total } \\
n=62\end{array}$} \\
\hline & & & & & & & \\
\hline \multirow{3}{*}{ Barreras } & Ninguna & $5 \%$ & $2,4 \%$ & $3,3 \%$ & $0 \%$ & $4,9 \%$ & $3,2 \%$ \\
\hline & 1 a 3 & $85 \%$ & $73,2 \%$ & $77 \%$ & $71,4 \%$ & $73,2 \%$ & $72,6 \%$ \\
\hline & 4a 6 & $10 \%$ & $24,4 \%$ & $19,3 \%$ & $28,6 \%$ & $22 \%$ & $24,2 \%$ \\
\hline \multirow{3}{*}{ Facilitadores } & Ninguna & & & & & & \\
\hline & 1 a 3 & $45 \%$ & $48,8 \%$ & $47,5 \%$ & $52,4 \%$ & $51,2 \%$ & $51,6 \%$ \\
\hline & 4a 6 & $55 \%$ & $51,2 \%$ & $52,5 \%$ & $47,6 \%$ & $48,8 \%$ & $48,4 \%$ \\
\hline \multirow{2}{*}{$\begin{array}{l}\text { Actitud hacia la consejería } \\
\text { en actividad física }\end{array}$} & No & $5 \%$ & $0 \%$ & $1,6 \%$ & & & \\
\hline & $\mathrm{Si}$ & $95 \%$ & $100 \%$ & $98,4 \%$ & $100 \%$ & $100 \%$ & $100 \%$ \\
\hline \multirow{2}{*}{$\begin{array}{l}\text { Conocimiento de las } \\
\text { recomendaciones en } \\
\text { actividad física }\end{array}$} & No hay & $7,7 \%$ & & $2,3 \%$ & $5,6 \%$ & $2,6 \%$ & $3,6 \%$ \\
\hline & Hay & $92,3 \%$ & $100 \%$ & $97,7 \%$ & $94,4 \%$ & $97,4 \%$ & $96,4 \%$ \\
\hline
\end{tabular}

Referente al cumplimiento de las recomendaciones de AF, se observó que el 39,3\% de los estudiantes de primer año cumplían con ellas $(45 \%$ de los hombres y $36,4 \%$ de las mujeres), y el $46.8 \%$ de los estudiantes (42,8\% hombres y 48,8\% mujeres) del otro grupo también lo hacían.

Sobre la realización de actividad física vigorosa se observó que en el primer año el 22,9\% cumplen con las recomendaciones (45\% de los hombres y $12,2 \%$ de las mujeres) así como el 21\% (19,1\% de los hombres y $21,9 \%$ de las mujeres) de quinto año, para la actividad física moderada, las recomendaciones fueron cumplidas por el 29,5\% y el $37,1 \%$, de los individuos de primero y quinto año, respectivamente Por último, se quiso indagar acerca de la actividad física global, en el grupo de primer año el 21,3\% de los estudiantes reportó no realizar ningún tipo de actividad física, en la misma categoría el $8,1 \%$ de los estudiantes reportó no realizar ningún tipo de actividad. (Tabla 3). 
Tabla 3. Distribución de los niveles de ejecución de actividad física según sexo en los dos grupos de estudiantes

\begin{tabular}{|c|c|c|c|c|c|c|c|}
\hline \multirow{4}{*}{ Característic } & 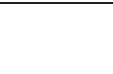 & \multirow{2}{*}{\multicolumn{3}{|c|}{$\begin{array}{c}\text { Primer Año } \\
(n=61) 49,6 \% \\
\text { Proporción según sexo }\end{array}$}} & \multirow{2}{*}{\multicolumn{3}{|c|}{$\begin{array}{c}\text { Quinto Año } \\
(n=62) 50,4 \% \\
\text { Proporción según sexo }\end{array}$}} \\
\hline & 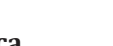 & & & & & & \\
\hline & & \multirow{2}{*}{ 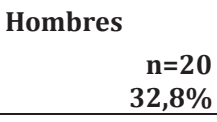 } & \multirow{2}{*}{$\begin{array}{r}\text { Mujeres } \\
\mathrm{n}=41 \\
67,2 \% \\
\end{array}$} & \multirow{2}{*}{$\begin{array}{l}\text { Total } \\
\qquad n=61\end{array}$} & \multirow{2}{*}{$\begin{array}{l}\text { Hombres } \\
\qquad \begin{array}{r}n=21 \\
33,9 \% \\
\end{array}\end{array}$} & \multirow{2}{*}{$\begin{array}{r}\text { Mujeres } \\
\mathrm{n}=41 \\
66,1 \% \\
\end{array}$} & \multirow{2}{*}{$\begin{array}{l}\text { Total } \\
n=62\end{array}$} \\
\hline & & & & & & & \\
\hline \multirow{4}{*}{$\begin{array}{c}\text { Nivel de actividad física } \\
\text { vigorosa }\end{array}$} & Inactivo & $40 \%$ & $61 \%$ & $54,1 \%$ & $23,8 \%$ & $41,5 \%$ & $35,5 \%$ \\
\hline & Bajo & $15 \%$ & $26,8 \%$ & $23 \%$ & $57,1 \%$ & $36,6 \%$ & $43,5 \%$ \\
\hline & Medio & $15 \%$ & $0 \%$ & $4,9 \%$ & $4,8 \%$ & $14,6 \%$ & $11,3 \%$ \\
\hline & Alto & $30 \%$ & $12,2 \%$ & $18 \%$ & $14,3 \%$ & $7,3 \%$ & $9,7 \%$ \\
\hline \multirow{4}{*}{$\begin{array}{l}\text { Nivel de actividad física } \\
\text { moderada }\end{array}$} & Inactivo & $25 \%$ & $26,8 \%$ & $26,2 \%$ & $4,8 \%$ & $12,2 \%$ & $9,7 \%$ \\
\hline & Bajo & $50 \%$ & $41,5 \%$ & $44,3 \%$ & $57,1 \%$ & $51,2 \%$ & $53,8 \%$ \\
\hline & Medio & $15 \%$ & $22 \%$ & $19,7 \%$ & $19 \%$ & $24,4 \%$ & $22,6 \%$ \\
\hline & Alto & $10 \%$ & $9,8 \%$ & $9,8 \%$ & $19 \%$ & $12,2 \%$ & $14,5 \%$ \\
\hline \multirow{4}{*}{$\begin{array}{l}\text { Nivel de cumplimiento } \\
\text { de las recomendaciones } \\
\text { de actividad física }\end{array}$} & Inactivo & $15 \%$ & $24,4 \%$ & $21,3 \%$ & $4,8 \%$ & $9,8 \%$ & $8,1 \%$ \\
\hline & Bajo & $40 \%$ & $39 \%$ & $39,3 \%$ & $52,4 \%$ & $41,5 \%$ & $45,2 \%$ \\
\hline & Medio & $15 \%$ & $24,4 \%$ & $21,3 \%$ & $19 \%$ & $24,4 \%$ & $22,6 \%$ \\
\hline & Alto & $30 \%$ & $12 \%$ & $18 \%$ & $23,8 \%$ & $24,4 \%$ & $24,2 \%$ \\
\hline
\end{tabular}

\section{Discusión}

La inactividad física (IF) es un problema de interés prioritario de salud pública, no solo por los efectos negativos sobre la salud y el bienestar de las personas, sino también por las consecuencias económicas que anualmente se le atribuyen. Según cifras publicadas en 2008, anualmente se estima que la IF le cuesta a los Estados Unidos alrededor de 50 billones de dólares al año.6,10 Las intervenciones de promoción de la $\mathrm{AF}$ pueden incrementar la expectativa de vida en 14,79 años, esto a su vez tiene un costo de 27,370 dólares por cada año de calidad de vida ganado.5,18

Acorde con esta evidencia, el tema de la promoción de la actividad física toma relevancia no solo a la hora de cuantificar los costos monetarios, sino también en salud de la población, los resultados observados en los grupos de estudiantes participantes en esta investigación son preocupantes, pues, los niveles de realización de AF reportados son inferiores a los observados en la población general, aun cuando, el currículo de la carrera de medicina incluye una asignatura obligatoria en el tema de actividad física y salud, en la que se les permite mejorar sus niveles de actividad física y alcanzar un mayor conocimiento sobre los beneficios de la misma en la salud de las personas.

En comparación con la población general, 39,3\% del total de estudiantes de primer año y $46,8 \%$ de quinto cumplieron o superaron la recomendación de realizar 150 minutos combinados o más de $\mathrm{AF}$ a la semana frente a $53,5 \%$ de la población general y según los datos reportados por otras facultades de medicina de Bogotá, se puede decir que los niveles de realización de AF son menores entre los estudiantes de la Universidad estudiada frente a las otras.15,16 Sin embargo, es interesante observar que los estudiantes de último año tienen niveles superiores de actividad física con respecto a sus pares de primero, contrario a lo observado en estudios de estudiantes de medicina en Colombia y pudiera mostrar algún efecto de la intervención en quinto semestre, aunque no es posible confirmarlo, debido a que no son los mismos estudiantes evaluados en el tiempo.

Respecto a las barreras para la práctica de $\mathrm{AF}$, se encontró que los estudiantes de quinto año reportaron tener más barreras y un menor 
porcentaje de facilitadores. En los dos grupos la mayor barrera enunciada fue la falta de tiempo, en concordancia con estudios enfocados en el tema de hábitos saludables en estudiantes de medicina en donde suelen atribuir a la falta de tiempo, la mayor dificultad para tener una vida saludable.12,14 El análisis de las barreras sugiere que en futuras intervenciones para mejorar los niveles de actividad física de los estudiantes, se debe pensar en la adjudicación de un tiempo para su práctica y en buscar una mayor participación de miembros de la comunidad universitaria, especialmente docentes, que permitan un soporte social más adecuado y coherente, así como pensar en actividades diferenciales.

En cuanto al conocimiento sobre actividad física, el número de respuestas acertadas en el último año fue menor y los estudiantes de último año reconocen que la facultad les ha proporcionado enseñanza sobre el tema, se requiere realizar un estudio enfocado en este ítem, puesto que en el presente, la aproximación es bastante general y debe ser refinada a través de investigaciones enfocadas en este tema que incluyan la construcción de instrumentos para tal fin.

Respecto a la actitud hacia la consejería en AF, se encontró que todos los estudiantes de quinto año, aparentemente presentan una actitud positiva hacia esta actividad, lo cual parece un resultado positivo, la AF la consideran un tema importante dentro de su formación y la valoran como parte importante en la promoción de salud, aunque también se considera que este resultado es descriptivo y debe ser evaluado por medio de estudios más específicos.

Los resultados de este estudio revelan que los estudiantes de quinto año presentan mayor sobrepeso y obesidad $(23,8 \%$ de los hombres y $9,8 \%$ de las mujeres), en comparación con sus pares de primer año. Estas proporciones son mayores a las observadas en un estudio realizado por otra universidad de la ciudad en el que se incluyeron estudiantes de ocho facultades de medicina, el cual reportó valores de 9\% y 13\% de los estudiantes de primer y quinto año, con sobrepeso y obesidad.14,15 En general, se ha observado que la población de estudiantes, puede presentar cifras menores en las proporciones de individuos con sobrepeso y/u obesidad, cuando es comparada con la población general de su mismo rango de edad.13,16

Si la educación médica tuviera éxito en la conciencia del estudiante, después de pasar por una carrera de medicina, se esperaría que la mayoría de los estudiantes fueran activos sin embargo, se ha visto que la realidad es otra. Basados en los resultados observados, se considera que los hábitos de los estudiantes deberían ser evaluados y que la construcción de sus propios hábitos saludables debería hacer parte de las competencias a adquirir en su proceso de formación profesional. Adicionalmente, la universidad debería propiciar condiciones de entorno adecuadas para alcanzar ese objetivo, de modo que el estudiante aprenda a partir de su propia construcción y vivencia, lo que podría redundar en la posibilidad futura de orientar mejor sobre el tema a sus pacientes.

El contar con dos muestras no seleccionadas aleatoriamente, de tamaño menor al que se requeriría para hacer análisis propios de la estadística inferencial y la administración del cuestionario en forma de autorreporte (lo que puede implicar algunas clases de sesgos), obligó a presentar solo resultados descriptivos, lo cual podría ser visto como una fuerte limitante del estudio, sin embargo, dado que no se cuenta con investigaciones previas de amplia envergadura en el tema en países latinoamericanos, se puede considerar que los resultados aquí presentados son de gran utilidad para ser usados como una línea de base en la exploración de un asunto que reviste gran importancia en la salud pública de los países.

\section{Agradecimientos}

Los datos para realizar este trabajo hacen parte del proyecto nacional liderado por la Universidad de los Andes denominado "Prevención de enfermedades crónicas: una prioridad en la formación médica" cuyo investigador principal es el Dr. John Duperly Sánchez a quien le agradecemos su gentileza.

Conflicto de intereses. Los autores no reportamos conflicto de intereses de ninguna clase. 


\section{Referencias}

1. Carter C, Hofer T, Seo A, Leeuwenburgh C. Molecular mechanisms of life-and health-span extension: role of calorie restriction and exercise intervention. Appl Physiol Nutr Metab. 2007;32(5):954-66.

2. Bazex J, Péne P, Riviére D. Physical activities and sport: Implications for health and society. Bull Acad Natl Med. 2012;196(7):1429-1442.

3. Angell S, Danel I, DeCock K. Global indicators and targets for noncommunicable diseases. Science. 2012; 337(6101):1456.

4. World Health Organization. Global status report on noncommunicable disease 2010. Geneve WHO. 2010; 2010.

5. Hallal P, Andersen L, Bull F, Guthold R, Haskell W, Ekelund U Series: Global physical activity levels: surveillance progress, pitfalls, and prospects. Lancet. 2012; 380(9838):247-57.

6. Bull F, Bauman A. Physical inactivity: The Cinderella risk factor for noncommunicable disease prevention. J Health Commun 2011; 16(Suppl. 2):13-26.

7. Luriatin A. Effective methods of losing weight in obesity. World experience. Med Tr Prom Ekol. 2013; 5(nd):4649.

8. Heath G, Parra D, Sarmiento O, Andersen L, Owen N, Goenka S, Montes F, Brownson R,. Evidence-based intervention in physical activity: lessons from around the world. Lancet. 2012; 380 (9838):272-81.

9. Ubirats E, Subirats Vila G, Soteras Martínez I. Prescripción de ejercicio físico: indicaciones, posología y efectos adversos. MedClin-Barcelona. 2012; 138(1):18-24.

10. Kohl HW 3rd1, Craig CL, Lambert EV, Inoue S, Alkandari $J R$, Leetongin $G$, et al. The pandemic of physical inactivity: global action for public health. Lancet. 2012; 380 (9838):294-305.

11. Oberg E, Frank E. Physicians' health practices strongly influence patient health practices. J R Coll Physicians Edinb. 2009;39(4):290-1.

12. Spencer E, Frank E, Elon L, Hertzberg V, Serdula M, Galuska D. Predictors of nutrition counseling behaviors and attitudes in US medical students. Am J Clin Nutr. 2006;84(3)655-662.

13. Frank E, Galuska D, Elon L, Wright E. Personal and clinical exercise-related attitudes and behaviors of freshmen U.S. medical students. Res Q Exerc Sport. 2004;75(2):112-21.

14. Contreras JJ, Espinoza RM, Dighero B, Drullinsky D, Liendo R, Soza F. Actitud sedentaria y factores asociados en estudiantes de Medicina. Rev Andal Med Deporte. 2009;2(4):133-40.

15. Duperly J, Lobelo F, Segura C, Sarmiento F, Herrera D, Frank E, et al. The association between colombian medical students' healthy personal habits and a positive attitude toward preventive counseling: cross-sectional analyses. BMC Public Health. 2009; 9:218.

16. Alba. L. Perfil de riesgo en estudiantes de Medicina de la Pontificia Universidad Javeriana. Univ. Med. 2009;50(2):143-155.
17. Duperly J, Sarmiento O, Parra D, Angarita C, Rivera D, Granados C, et al. Documento técnico con los contenidos de direccionamiento pedagógico para la promoción de hábitos de vida saludable, con énfasis en alimentación saludable y el fomento de ambientes $100 \%$ libres de humo de cigarrillo a través de la práctica regular de la actividad física cotidiana, dirigidos a los referentes de las entidades territoriales. Ministerio de la Protección Social, COLDEPORTES Imprenta Nacional, BogotáColombia. 2011.

18. Frank E, Carrera JS, Elon L, Hertzberg VS. Predictors of US medical students' prevention counseling practices. Prev Med. 2007;44:76-81.

19. Colombia. Ministerio de la Protección Social. Encuesta Nacional de la Situación Nutricional en Colombia 2010, Bogotá; 2011. 